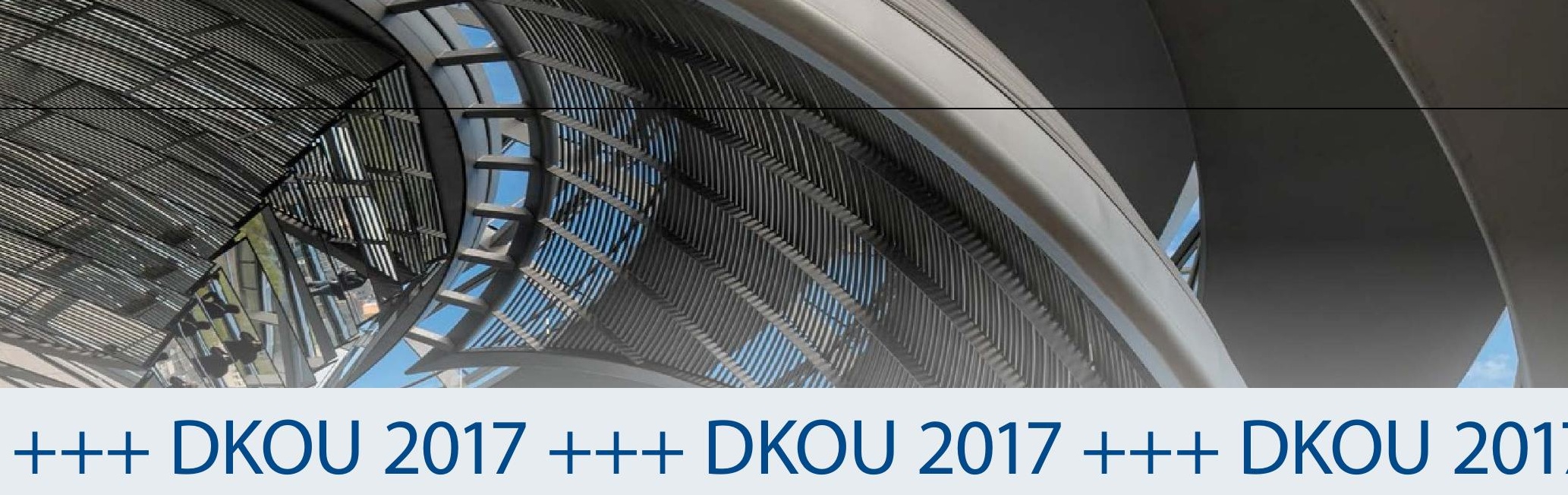

\section{Distale Humerusfraktur im Alter: wie vorgehen?}

D ie extraartikuläre Fraktur des distalen Humerus ist eine typische Fraktur älterer Frauen ( $>75$ Jahre) nach einem Sturz auf die ausgestreckte Hand. Wann rekonstruiert, wann endoprothetisch versorgt werden sollte, war Thema der Sitzung ,Kontroversen in der Unfallchirurgie“. Gerade im Hinblick auf die häufig für die Mobilität wichtige Nutzung eines Rollators sprach sich Prof. Wolfgang Lehmann, Direktor der Klinik für Unfallchirurgie, Orthopädie und plastische Chirurgie der Universität Göttingen, für eine Rekonstruktion aus. Der zeitliche Aufwand lohne sich, denn mit den heutigen Osteosynthesen lasse sich sogar bei mehrteiliger Fraktur ein stabiles Ellbogengelenk auch bei voller Kraftanwendung erzielen. Nach einer endoprothetischen Versorgung sei die Belastungsfähigkeit hingegen dauerhaft eingeschränkt. Als weitere Risiken der Prothesenversorgung nannte Lehmann die Gefahr von Nervenschädigungen, Gelenkinfektionen, Ossifikationen und Abscherungen aufgrund der oft osteoporotischen Knochensubstanz, die Reoperationen erforderlich machen können.
Als Standard gelten heute präformierte Doppelplattenosteosynthesen mit multidirektionaler, winkelstabiler Verblockung. Dabei mache es im Hinblick auf das biomechanische und klinische Ergebnis keinen Unterschied, ob die Frakturen orthogonal oder parallel verplattet würden, so Lehmann. Unabhängig von der Knochenqualität sei eine winkelstabile Fixierung auch bei multiplen Fragmenten möglich. Lehmann plädierte dafür, eine primäre Implantation einer Ellbogengelenkprothese nur bei fehlender Rekonstruktionsmöglichkeit, etwa bei Abscherung oder Zertrümmerung des Capitulums, und als Rettungsstrategie bei nicht verbesserbarer, fehlgeschlagener Osteosynthese zu erwägen.

Auch Prof. Lars-Peter Müller, Direktor der Klinik und Poliklinik für Orthopädie und Unfallchirurgie, Köln, betonte das häufig schlechte Ergebnis bei endoprothetischer Versorgung des Ellbogengelenks in der Traumasituation. Die Komplikationsrate sei sehr viel höher als bei Patienten mit rheumatoider Arthritis und betrage nach einer Studie $57 \%$ nach
6,1 Jahren. Dennoch sei es wichtig, bei über 65-Jährigen nach einem Ellenbogentrauma die Entscheidung zur Prothesenversorgung primär und nicht erst sekundär zu treffen. Denn die Erfolgschancen seien noch geringer, wenn der Nervus ulnaris und der Musculus trizeps bereits vorpräpariert seien. Gleichzeitig zeige eine zwar ältere, aber relevante Studie, dass auch die sekundäre Versorgung mit einer Totalendoprothese (TEP) ein gangbarer Weg sei, weil Patientenzufriedenheit und Ergebnis sich kaum gegenüber der primären TEP unterscheiden. Bei tiefer distaler Humerusfraktur, also unterhalb der Fossa verlaufender Bruchlinie, plädierte Müller in jedem Fall für eine primäre Endoprothese. Dabei müsse der Trizeps nicht abgelöst werden. Durch das sogenannte Triceps-on-Vorgehen lasse sich die Komplikationsrate senken. Da die Ulna dabei aber nicht so gut einsehbar sei, bestehe allerdings ein gewisses Risiko einer Perforation. Von einer Hemiendoprothese riet Müller ab, weil sie ungekoppelt und es somit schwieriger sei, stabile Gelenkverhältnisse zu erzeugen. Zudem müssten dafür die Bänder intakt sein und nicht zuletzt dauere die Operation länger.

\title{
Neues zur Stoßwellentherapie
}

$\mathrm{E}_{\mathrm{s} \text { side }}$ rgebnisse zu alten und neuen Indikati-onen zur extrakorporalen Stoßwellentherapie stellte PD Dr. Jörg Hausdorf, Oberarzt an der Klinik für Orthopädie, Physikalische Medizin und Rehabilitation, Klinikum der Universität München, Campus Großhadern, vor. Als wichtigste Indikation erachtet er weiterhin die Fasziitis plantaris, und zwar nicht nur in der chronischen Situation, sondern auch bei akuten Beschwerden. Wie eine aktuelle
Studie zeigt, ist es nicht erforderlich, den Kalk zuvor mittels Ultraschall zu lokalisieren. Genauso gut kann dort geschallt werden, wo es weh tut. Auch für die bewährte Indikation der Epikondylitis gibt es Neuigkeiten: Die Behandlung mit $3 \times 1.000$ Impulsen niedriger Energie $(0,10$ $\mathrm{mJ} / \mathrm{mm}^{2}$ ) erzielte nach zwölf Wochen bessere Ergebnisse als $3 \times 1.000$ Impulse mit $0,25 \mathrm{~mJ} / \mathrm{mm}^{2}$. Auch bei der Rotatorenmanschetten-Tendopathie ohne Kalk lässt sich durch eine Stoßwellentherapie eine signifikante Schmerzreduktion erzielen. Bei einem subachromialen Schmerzsyndrom mit/ohne Kalk ist durch die gezielte Behandlung einzelner Sehnen kein Zusatznutzen erreichbar, ebensowenig durch eine zusätzliche exzentrische Trainingstherapie. Effektiv ist die Stoßwellentherapie Hausdorf zufolge auch bei der Achillessehnenentzündung. Potenzial besitzt das Verfahren auch bei schmerzhaften Knochenmarködemen, wo es die Rückbildung unterstützt.

kat 